\title{
Discharge plans to prevent hospital readmission for acute exacerbations in children with chronic respiratory illness (Protocol)
}

Hall KK, Chang AB, O'Grady KF

Hall KK, Chang AB, O'Grady KF.

Discharge plans to prevent hospital readmission for acute exacerbations in children with chronic respiratory illness.

Cochrane Database of Systematic Reviews 2016, Issue 8. Art. No.: CD012315.

DOI: 10.1002/14651858.CD012315.

www.cochranelibrary.com 
TABLE OF CONTENTS

HEADER 1

ABSTRACT

BACKGROUND

OBJECTIVES

METHODS

ACKNOWLEDGEMENTS

REFERENCES

APPENDICES

CONTRIBUTIONS OF AUTHORS

DECLARATIONS OF INTEREST

SOURCES OF SUPPORT 
[Intervention Protocol]

\section{Discharge plans to prevent hospital readmission for acute exacerbations in children with chronic respiratory illness}

Kerry K Hall ${ }^{1}$, Anne B Chang ${ }^{1,2}$, KerryAnn F O'Grady ${ }^{1}$

1Institute of Health and Biomedical Innovation, Queensland University of Technology, Brisbane, Australia. ${ }^{2}$ Child Health Division, Menzies School of Health Research, Charles Darwin University, Darwin, Australia

Contact address: Kerry K Hall, Institute of Health and Biomedical Innovation, Queensland University of Technology, 62 Graham Street, South Brisbane, Brisbane, Queensland, 4101, Australia. kerry.hall@hdr.qut.edu.au, kkmjhall@bigpond.com.

Editorial group: Cochrane Airways Group

Publication status and date: Edited (no change to conclusions), published in Issue 8, 2016.

Citation: Hall KK, Chang AB, O'Grady KF. Discharge plans to prevent hospital readmission for acute exacerbations in children with chronic respiratory illness. Cochrane Database of Systematic Reviews 2016, Issue 8. Art. No.: CD012315. DOI: 10.1002/14651858.CD012315.

Copyright (c) 2016 The Cochrane Collaboration. Published by John Wiley \& Sons, Ltd.

\section{A B S T R A C T}

This is a protocol for a Cochrane Review (Intervention). The objectives are as follows:

To evaluate the efficacy of individual caseworker-assigned discharge plans, compared to non-caseworker-assigned plans, in preventing hospitalisation for acute respiratory exacerbations in children with chronic lung diseases such as asthma and bronchiectasis. 


\section{B A C K G R O U N D}

\section{Description of the condition}

Chronic respiratory conditions are major causes of mortality and morbidity in developed and developing countries. Children with chronic health conditions have increased morbidity associated with their physical, emotional and general well-being. Their illness and these stressors can have a great impact on parents and caregivers and their ability to cope with ongoing care, especially when recurrent hospitalisations are required (Peterson-Carmichael 2012; Plant 2013). Disability from chronic diseases is increasing as the burden from acute infectious diseases is being controlled through treatment and prevention (Ait-Khaled 2001; Asher 2014; Beran 2015; Chang 2013). Chronic respiratory diseases that are important in childhood include recurrent protracted bacterial bronchitis (PBB), asthma, chronic suppurative lung disease, and bronchiectasis (Chang 2014). These diseases present challenges to public health in both developed and developing countries alike because of their high prevalence, the burden they place on the individual and the high economic cost to health systems globally (Abramson 2015; Asher 2014; Chang 2013; Kapur 2010; Zar 2014). Furthermore, repeated exacerbations of chronic lung diseases in childhood (e.g. bronchiectasis) are known precursors to further lung function decline (Kapur 2010).

Acute respiratory exacerbations (AREs) are common in children with chronic respiratory disease, often requiring admission to hospital (Redding 2014). A retrospective study in Brisbane, Australia, of children with non-cystic fibrosis bronchiectasis reported that $35 \%$ of AREs required hospitalisation (Kapur 2009). A multicentre RCT of Indigenous children in Australia and Alaska found that $15 \%$ of children were hospitalised for an ARE during the study period, and that $52 \%$ of children at enrolment to the RCT had been hospitalised for an ARE in the previous 12 months before enrolment (Redding 2014). A retrospective, cross-sectional study from the United States of America (USA) reported hospital admission rates of $18 \%$ in children with asthma exacerbations (Sanders 2007). Reducing the frequency of AREs and recurrent hospitalisations is therefore an important goal in the individual and public health management of chronic respiratory illnesses in children. Discharge planning is a process used to decide what a person needs for transition from one level of care to another and is usually considered in the context of discharge from hospital to the home. Historically discharge planning from hospital for ongoing management of an illness has been referral to a GP or allied health professional or self-management by the individual and their family with limited communication between the hospital and patient once discharged. Effective discharge planning can decrease the risk of recurrent acute exacerbations of chronic disease with or without readmission to hospital (Lorig 2003).

In this review we will focus on children who have been hospitalised with AREs with or without deterioration of the following chronic respiratory conditions: asthma; recurrent protracted bacterial bronchitis; chronic suppurative lung disease; and bronchiectasis. The aim of the review is to determine the effectiveness of individual, caseworker-assigned discharge planning and followup, during and after hospitalisation, in reducing subsequent hospital admissions in children with chronic respiratory diseases compared to traditional discharge-planning approaches (including self-management).

\section{Description of the intervention}

Traditional hospital-discharge plans tend to focus on diseasespecific information and skills required to manage the disease, for example blood glucose testing in diabetes. The information can be written, verbal or both and is usually delivered to the patient by a ward nurse. A discharge summary of the hospital stay is usually sent to the patient's general practitioner (GP) and can include diagnostic findings and post-discharge follow-up requirements (Kripalani 2007). Discharge plans can vary in health facilities and across cultures (Holland 2007). Traditional discharge plans are often based on the medical management of the condition and may not consider other aspects of living that are impacted by chronic illness such as the emotional well-being of the individual, their support network and the general tasks of daily living (Lorig 2003).

Self-management plans are formalised care plans developed while the person is in hospital that aims to teach them and their family/carer the required skills to manage and control their health condition. Self-management plans teach strategies to control disease, promote health and how to live with a chronic health condition. This generally includes monitoring and managing symptoms and signs of illness, managing the impacts of illness and adhering to treatment regimens which allow the person to identify changes in their condition and to implement appropriate changes as their condition or disease changes (Audulv 2013; Lorig 2003; Regan-Smith 2006). Self-management discharge plans don't generally incorporate follow-up post hospital discharge by the discharge planner to determine plan outcomes. Self management can be effective if the patient and his/her family have the motivation and confidence to self manage their illness. Self management plans can fail if considerations have not been given to the patient's perception of health care practices and cultural considerations. For self-management plans to be effective the individual, carer and primary health care provider need to continually engage and be proactive in the health care plan, which can be difficult for parents of chronically ill children (Lorig 2003).

Individual caseworker-assigned plans involve the patient being assigned a specific caseworker on admission, or shortly after, and aim to address the gaps in simple or self-management discharge plans described above. The caseworker's role is to liaise with hospital staff during the person's stay in hospital and develop a plan for discharge which provides individual, ongoing support to patients and their families to improve health outcomes.This may be done by support and motivation to attend appointments and behaviour risk modification through education programmes (Plant 2013). A Cochrane review that included generic discharge planning from hospital found that tailored discharge planning led to a "small reduction in hospital length of stay and reduced the risk of readmission to hospital at three months follow-up for older people with a medical condition" but did not reduce costs to the health service (Gonçalves-Bradley 2016). This review did not specifically examine individualised caseworker-assigned plans that incorporate one-on-one care post discharge until resolution of symptoms or care is no longer required (Gonçalves-Bradley 2016). Individualised caseworker plans are likely to be particularly important in settings where a person with low health literacy or from a different culture requires assistance to navigate the health system. A Cochrane review on Indigenous health worker involvement (compared to routine care) for Indigenous people 
with asthma described improvements in knowledge scores but no significant difference in exacerbation rates (Chang 2010).

\section{How the intervention might work}

Caseworkers may reduce re-hospitalisation of people with chronic conditions through a variety of ways. These can include: (a) improving communication between service providers and patients hence leading to better self-management plans; (b) understanding the types of available inpatient and outpatient hospital services so as to maximise outpatient care; (c) coordinating care from hospital to home and beyond; and (d) improved health education and promotion such as reducing tobacco smoking exposure which will reduce acute exacerbations of chronic diseases (Jordan 2007; Plant 2013). A holistic approach to chronic disease management may provide opportunities to identify early barriers to full recovery and early symptom management could prevent deterioration in the person's condition that, if not addressed, would be likely to result in readmission to hospital.

\section{Why it is important to do this review}

The morbidity (including hospitalisations) related to chronic respiratory disease is an important issue for patients and health systems globally. Preventing possible triggers (e.g. infections, poor adherence to medications, etc) that could cause exacerbations leading to hospitalisation have important individual and public health impacts (Ait-Khaled 2001; Chang 2013; Plant 2013). The appropriate management and follow-up of children post-discharge through individualised case management is one possible strategy that may reduce the overall burden of disease and long-term sequelae of chronic respiratory disease such as bronchiectasis. However, this strategy would likely add a cost to the health system. A systematic review of individual caseworker-assigned discharge care plans (versus standard care) to determine the effectiveness of this strategy in reducing the hospitalisations and the burden of disease in children would inform clinical care and health policy.

\section{O B JECTIVES}

To evaluate the efficacy of individual caseworker-assigned discharge plans, compared to non-caseworker-assigned plans, in preventing hospitalisation for acute respiratory exacerbations in children with chronic lung diseases such as asthma and bronchiectasis.

\section{METHODS}

\section{Criteria for considering studies for this review \\ Types of studies}

We will include randomised controlled trials (RCTs) (including cluster randomised trials). We will include studies reported as full text, those published as abstract only and unpublished data. Crossover trials will not be included.

\section{Types of participants}

We will include children aged less than 18 years admitted to hospital with an acute respiratory exacerbation or deterioration of an underlying chronic respiratory illness.

Exclusion criteria: diagnosis of cystic fibrosis.

\section{Types of interventions}

We will describe individual caseworker-assigned plans as those involving the caseworker facilitating communication on admission to the hospital setting between the child, family and attending physician as well as the caseworker involving the child and family in developing and implementing the plan of care during and following hospitalisation. Caseworker activities could include, but not be limited to: a) facilitating the discharge plan and obtaining needed consultations from other allied health services as required; b) collaborating with home health agencies; and c) providing educational information and emotional support to the child and family.

We will include studies comparing caseworker-assigned discharge plans versus discharge plans that do not involve caseworker support.

\section{Types of outcome measures}

\section{Primary outcomes}

1. Rate and frequency of exacerbations requiring emergency department visit or hospitalisation

2. Adverse events (all causes)

\section{Secondary outcomes}

1. Rate of unscheduled healthcare visits to a GP

2. Quality of life (measured on a validated scale) at months one and six post-discharge

3. Cost-effectiveness

4. Duration of stay at subsequent hospitalisations

5. Adherance to discharge medications

6. Mortality rate post-discharge for respiratory related illness

\section{Search methods for identification of studies}

\section{Electronic searches}

We will identify studies from searches of the following databases:

- The Cochrane Airways Group Register of Trials - all years

- Cochrane Central Register of Controlled Trials (CENTRAL), latest issue (The Cochrane Library)

- MEDLINE (Ovid) 1950 to date

- EMBASE (Ovid) 1974 to date

- Trials registries (ClinicalTrials.gov and the World Health Organization (WHO) trials portal (apps.who.int/trialsearch/))

The proposed MEDLINE strategy is listed in Appendix 1. This will be adapted for use in the other databases. All databases will be searched from their inception to the present, and there will be no restriction on language of publication. We will handsearch conference abstracts and will search for grey literature through the CENTRAL database.

\section{Searching other resources}

We will check the reference lists of all primary studies and review articles for additional references. We will search the references from identified papers and reviews for further references and we will contact authors to request their identification of any unpublished or missed trials. 
We will search for errata or retractions from included studies published in full-text on PubMed (www.ncbi.nlm.nih.gov/pubmed) and report the date this was done within the review.

\section{Data collection and analysis}

\section{Selection of studies}

Two review authors ( $\mathrm{KH}$ and $\mathrm{KO}$ ) will independently screen titles and abstracts of all the potential studies we identify as a result of the search and code them as 'retrieve' (eligible or potentially eligible/unclear) or 'do not retrieve'. We will retrieve the fulltext study reports/publications and two review authors $(\mathrm{KH}$ and $\mathrm{KO}$ ) will independently screen the full-text and identify studies for inclusion, and identify and record reasons for exclusion of the ineligible studies. We will resolve any disagreement through discussion or, if required, we will consult a third review author (AC). We will identify and exclude duplicates and collate multiple reports of the same study so that each study rather than each report is the unit of interest in the review. We will record the selection process in sufficient detail to complete a PRISMA flow diagram and 'Characteristics of excluded studies' table (Moher 2009). While not part of the quantitative analyses, we also plan a qualitative synthesis of findings from non-RCTs addressing the role of caseworker-assigned discharge management strategies. Those findings will be addressed in the discussion to provide an overview of the evidence to support this strategy for reducing recurrent AREs in children with chronic respiratory diseases.

\section{Data extraction and management}

We will use a data collection form for study characteristics and outcome data which has been piloted on at least one study in the review. Two review authors ( $\mathrm{KH}$ and $\mathrm{KO}$ ) will extract study characteristics from included studies. We will extract the following study characteristics:

1. Methods: study design, total duration of study, details of any 'run in' period, number of study centres and location, study setting, withdrawals, and date of study.

2. Participants: number, mean age, age range, gender, indigenous status, socio-economic status, diagnosis, severity of condition, diagnostic criteria, inclusion criteria, and exclusion criteria.

3. Interventions: intervention, comparison, concomitant medications, and excluded medications.

4. Outcomes: primary and secondary outcomes specified and collected, and time points reported.

5. Notes: funding for trial, and notable conflicts of interest of trial authors.

Two review authors ( $\mathrm{KH}$ and $\mathrm{KO}$ ) will independently extract outcome data from the included studies. We will note in the 'Characteristics of included studies' table if outcome data were not reported in a usable way. We will resolve disagreements by consensus or by involving a third review author (AC). One review author $(\mathrm{KH})$ will transfer data into the Review Manager file (RevMan 2014). We will double-check that data are entered correctly by comparing the data presented in the systematic review with the study reports. A second review author (KO) will spot-check study characteristics for accuracy against the trial report.

\section{Assessment of risk of bias in included studies}

Two review authors ( $\mathrm{KH}$ and $\mathrm{KO}$ ) will independently assess risk of bias for each study using the criteria outlined in the Cochrane Handbook for Systematic Reviews of Interventions (Higgins 2011). We will resolve any disagreements by discussion or by involving another review author (AC). We will assess the risk of bias according to the following domains:

1. Random sequence generation.

2. Allocation concealment.

3. Blinding of participants and personnel.

4. Blinding of outcome assessment.

5. Incomplete outcome data.

6. Selective outcome reporting.

7. Other bias.

We will grade each potential source of bias as high, low or unclear and provide a quote from the study report together with a justification for our judgement in the 'Risk of bias' table. We will summarise the risk of bias judgements across different studies for each of the domains listed. We will consider blinding separately for different key outcomes where necessary (e.g. for unblinded outcome assessment, risk of bias for all-cause mortality may be very different than for a patient-reported pain scale). Where information on risk of bias relates to unpublished data or correspondence with a trialist, we will note this in the 'Risk of bias' table.

When considering treatment effects, we will take into account the risk of bias for the studies that contribute to that outcome.

\section{Assessment of bias in conducting the systematic review}

We will conduct the review according to this published protocol and report any deviations from it in the 'Differences between protocol and review' section of the systematic review.

\section{Measures of treatment effect}

We will analyse dichotomous data as odds ratios (OR) and continuous data as mean differences or standardised mean differences. We will enter data presented as a scale with a consistent direction of effect.

We will undertake meta-analyses only where this is meaningful i.e. if the treatments, participants and the underlying clinical question are similar enough for pooling to make sense.

We will narratively describe skewed data reported as medians and interquartile ranges.

Where multiple trial arms are reported in a single trial, we will include only the relevant arms. If two comparisons (e.g. intervention $A$ versus control and intervention $B$ versus control) are combined in the same meta-analysis, we will halve the control group to avoid double-counting.

\section{Unit of analysis issues}

For dichotomous data, we will report the proportion of participants contributing to each outcome in comparison with the total number randomised. For rate ratios of common events whereby one participant may have more than one event, we will use generic 
inverse variance (GIV). The rate ratios will be taken from the published papers and the standard error of the log rate ratios calculated from confidence intervals or $\mathrm{P}$ values published in the papers.

\section{Dealing with missing data}

We will contact investigators or study sponsors in order to verify key study characteristics and obtain missing numerical outcome data where possible (e.g. when a study is identified as abstract only). Where this is not possible, and the missing data are thought to introduce serious bias, we will explore the impact of including such studies in the overall assessment of results by a sensitivity analysis.

\section{Assessment of heterogeneity}

We will use the $I^{2}$ statistic to measure heterogeneity among the studies in each analysis. If we identify substantial heterogeneity we will report it and explore possible causes by prespecified subgroup analysis.

\section{Assessment of reporting biases}

If we are able to pool more than 10 studies, we will create and examine a funnel plot to explore possible small study and publication biases.

\section{Data synthesis}

We will use the random-effects model analyses as we expect variations in effects due to the differences in study populations and interventions. We will perform a sensitivity analysis with a fixed-effect model. We will determine the summary OR and mean differences with their 95\% confidence intervals (CI) using a metaregression model. If there are sufficient studies for the analysis, we will use a random-effects model whenever there are concerns about statistical heterogeneity.

\section{Summary of findings table}

We will create a 'Summary of findings' table using the following outcomes.

1. Rate and frequency of exacerbations requiring emergency department visit or hospitalisation

2. Rate of unscheduled healthcare visits to a GP

3. Quality of life (measured on a validated scale) at months one and six post-discharge

4. Cost-effectiveness

5. Duration of stay at subsequent hospitalisations

6. Adherance to discharge medications

7. Mortality rate post-discharge for respiratory-related illness

We will use the five GRADE considerations (study limitations; consistency of effect; imprecision; indirectness; and publication bias) to assess the quality of a body of evidence as it relates to the studies which contribute data to the meta-analyses for the prespecified outcomes. We will use methods and recommendations described in Section 8.5 and Chapter 12 of the Cochrane Handbook for Systematic Reviews of Interventions (Higgins 2011) using GRADEpro software. We will justify all decisions to down- or up-grade the quality of studies using footnotes and we will make comments to aid the reader's understanding of the review where necessary.

\section{Subgroup analysis and investigation of heterogeneity}

We plan to carry out the following subgroup analyses.

1. Disease types: chronic suppurative lung disease, bronchiectasis, asthma, others

2. Two or more co-morbidities - these co-morbidities may include but not be limited to the following: atopy, dysphagia, bronchopulmonary dysplasia, juvenile diabetes, congenital/ neurological disorders, obstructive sleep apnoea

3. Age groups of children: pre-school (aged $<6$ years) versus older children (aged 6 or more years)

4. Control group (e.g. usual discharge letter, action plans)

5. Setting: low income economies, low-middle income economies, upper-middle income economies, high income economies, high income OECD members

6. Indigenous versus non-indigenous

7. Hospital type e.g. tertiary paediatric referral centre or local/ district hospital

We will use the formal test for subgroup interactions in Review Manager 5 (RevMan 2014).

\section{Sensitivity analysis}

We plan to carry out the following sensitivity analyses, excluding the following from primary analyses.

1. Studies at high risk of bias detection.

2. Unpublished data (obtained from study authors or from conference abstracts).

We will compare the results from a fixed-effect model as opposed to the random-effects model.

\section{ACKNOWLEDGEMENTS}

Sean Beggs was the Contact Editor for this protocol and commented critically upon the document.

The Background and Methods sections of this protocol are based on a standard template used by Cochrane Airways.

This project was supported by the National Institute for Health Research (NIHR), via Cochrane Infrastructure funding to Cochrane Airways. The views and opinions expressed therein are those of the authors and do not necessarily reflect those of the Systematic Reviews Programme, NIHR, NHS or the Department of Health. 


\section{REFERE N CES}

\section{Additional references}

\section{Abramson 2015}

Abramson MJ, Koplin J, Hoy R, Dharmage SC. Population-wide preventive interventions for reducing the burden of chronic respiratory disease. International Journal of Tuberculosis Lung Disease September 2015;19(9):1007-18.

\section{Ait-Khaled 2001}

Ait-Khaled N, Enarson D, Bousquet J. Chronic respiratory diseases in developing countries: the burden and strategies for prevention and management. Bulletin of World Health Organization November 2001;79(10):971-9.

\section{Asher 2014}

Asher I, Pearce N. Global burden of asthma among children. International Journal of Tuberculosis and Lung Disease November 2014;18(11):1269-78.

\section{Audulv 2013}

Audulv A. The over time development of chronic illness selfmanagement patterns: A longitudinal qualitative study. $B M C$ Public Health 2013;13:452.

\section{Beran 2015}

Beran D, Zar HJ, Perrin C, Menezes AM, Burney P, Forum of International Respiratory Societies working group collaboration. Burden of asthma and chronic obstructive pulmonary disease and access to essential medicines in lowincome and middle-income countries. The Lancet Respiratory Medicine February;3(2):159-70.

\section{Chang 2010}

Chang AB, Taylor B, Masters IB, Laifoo Y, Brown ADH. Indigenous healthcare worker involvement for Indigenous adults and children with asthma. Cochrane Database of Systematic Reviews 2010, Issue 5. [DOI: 10.1002/14651858.CD006344.pub3]

\section{Chang 2013}

Chang LH, Rivera MP. Respiratory diseases: meeting the challenges of screening, prevention, and treatment. North Carolina Medical Journal 2013;74(5):385-92.

\section{Chang 2014}

Chang AB, Brown N, Toombs M, Marsh RL, Redding GJ. Lung disease in indigenous children. Paediatric Respiratory Reviews 2014;15(4):325-32.

\section{Gonçalves-Bradley 2016}

Gonçalves-Bradley DC, Lannin NA, Clemson LM, Cameron ID, Shepperd S. Discharge planning from hospital. Cochrane Database of Systematic Reviews 2016, Issue 1. [DOI: 10.1002/14651858.CD000313.pub5]

\section{Higgins 2011}

Higgins JPT, Green S (editors). Cochrane Handbook for Systematic Reviews of Interventions Version 5.1.0 [updated March 2011]. The Cochrane Collaboration, 2011. Available from www.cochrane-handbook.org.

\section{Holland 2007}

Holland DE, Harris MR. Discharge planning, transitional care, coordination of care, and continuity of care: Clarifying concepts and terms from the hospital perspective. Home Health Care Services Quarterly 2008;26(4):3-19.

\section{Jordan 2007}

Jordan JE, Osborne RH. Chronic disease self-management education programs: challenges ahead. Medical Journal of Australia 2007;186(2):84-7.

\section{Kapur 2009}

Kapur N, Masters IB, Chang AB. Exacerbations in non-cystic fibrosis bronchiectasis: Clinical features and investigations. Respiratory Medicine 2009;103(11):1681-7.

\section{Kapur 2010}

Kapur N, Masters IB, Chang AB. Longitudinal growth and lung function in paediatric non-cystic fibrosis bronchiectasis: what influences lung function stability?. Chest 2010;138(1):158-64.

\section{Kripalani 2007}

Kripalani S, LeFevre F, Phillips CO, Williams MV, Basaviah P, Baker DW. Deficits in communication and information transfer between hospital-based and primary care physicians: Implications for patient safety and continuity of care. JAMA 2007;297(8):831-41.

\section{Lorig 2003}

Lorig KR, Holman HR. Self-management education: History, definition, outcomes, and mechanisms. Annals of Behavioral Medicine 2003;26(1):1-7.

\section{Moher 2009}

Moher D, Liberati A, Tetzlaff J, Altman DG, The Prisma Group. Preferred Reporting Items for Systematic Reviews and Meta-Analyses: The PRISMA Statement. PLoS Medicine 2009;6(7):264-70

\section{Peterson-Carmichael 2012}

Peterson-Carmichael SL, Cheifetz IM. The chronically critically ill patient: paediatric considerations. Respiratory Care 2012;57(6):993-1003.

\section{Plant 2013}

Plant N, Mallitt K-A, Kelly PJ, Usherwood T, Gillespie J, Boyages S, et al. Implementation and effectiveness of 'care navigation', coordinated management for people with complex chronic illness: rationale and methods of a randomised controlled. BMC Health Services Research 2013;13(1):1-6.

\section{Redding 2014}

Redding GJ, Singleton RJ, Valery PC, Williams H, Grimwood K, Morris PS, et al. Respiratory exacerbations in indigenous children from two countries with non-cystic fibrosis chronic suppurative lung disease/bronchiectasis. Chest 2014;146(3):762-74 


\section{Regan-Smith 2006}

Regan-Smith M, Hirschmann K, Lobst W, Battersby M. Teaching residents chronic disease management using the Flinders Model. Journal of Cancer Education 2006;21(2):60-2.

\section{RevMan 2014 [Computer program]}

The Nordic Cochrane Centre, The Cochrane Collaboration. Review Manager (RevMan). Version 5.3. Copenhagen: The Nordic Cochrane Centre, The Cochrane Collaboration, 2014.

\section{Sanders 2007}

Sanders DL, Gregg W, Aronsky D. Identifying asthma exacerbations in a paediatric emergency department: a feasibility study. International Journal of Medical Informatics 2007;76(7):557-64.

\section{Zar 2014}

Zar HJ, Ferkol TW. The global burden of respiratory diseaseimpact on child health. Pediatric Pulmonology 2014;49(5):430-4.

\section{APPENDICES}

\section{Appendix 1. Search strategies}

\section{MEDLINE (Ovid)}

1. $\exp$ Asthma/

2. asthma\$.tw.

3. Respiratory Sounds/

4. wheez\$.tw.

5. exp Bronchiectasis/

6. bronchiectasis\$.tw.

7. Lung Diseases/

8. exp Suppuration/

9. (chronic\$ adj3 suppur\$ adj3 lung\$).tw.

10. exp Pneumonia/

11. (recur\$ adj2 pneumonia\$).tw.

12. exp Bronchitis/

13. ((bacterial\$ or recur\$) adj2 bronchitis\$).tw.

14. ((chronic\$ or recurrent\$) adj2 (respiratory\$ or lung\$) adj2 (illness or disease\$)).tw.

15. or/1-14

16. $\exp$ Child/

17. exp Pediatrics/

18. exp infant/

19. exp adolescent/

20. (paediatric\$ or pediatric\$ or child\$ or adolescen\$ or infant\$ or young\$ or preschool\$ or pre-school\$ or newborn\$ or new-born\$ or neonat\$ or neo-nat\$).tw.

21. or $/ 16-20$

22. 15 and 21

23. Patient Discharge/

24. Patient Care Planning/

25. Case Management/

26. (discharge\$ adj3 (plan\$ or program\$ or intervention\$ or service or procedure\$)).tw.

Discharge plans to prevent hospital readmission for acute exacerbations in children with chronic respiratory illness (Protocol) 
27. ((patient\$ or hospital\$) adj2 discharge\$).tw.

28. or $/ 23-27$

29. 22 and 28

30. (controlled clinical trial or randomized controlled trial).pt.

31. (randomized or randomised).ab,ti.

32. placebo.ab,ti.

33. dt.fs.

34. randomly.ab,ti.

35. trial.ab,ti.

36. groups.ab,ti.

37. or/30-36

38. Animals/

39. Humans/

40. 38 not (38 and 39)

41.37 not 40

42. 29 and 41

\section{CONTRIBUTIONS OF AUTHORS}

$\mathrm{KH}$ predominantly described the background and was responsible for formatting. KO predominantly described the data analysis strategy. $\mathrm{KH}, \mathrm{KO}$ and $\mathrm{AC}$ contributed to the body of methodology. $\mathrm{KH}$ and $\mathrm{KO}$ will independently extract outcome data from the included studies for the review. KH will transfer data into Review Manager, KO will check transferred data for accuracy. AC will provide clinical expertise and has provided guidance with protocol development and edited the protocol. All authors approved the final draft before submission.

\section{DECLARATIONS OF INTEREST}

$\mathrm{KH}:$ no interests to declare.

KO: no interests to declare.

AC: no interests to declare.

\section{SOURCES OF SUPPORT}

\section{Internal sources}

- The authors declare that no such funding was received for this systematic review, Other.

\section{External sources}

- National Health and Medical Research Council Centre, Australia.

A top-scholarship provided to Kerry Hall by the NHMRC Centre for Research Excellence in Lung Health for Aboriginal and Torres Strait Islander Children

- Australian Government, Australia.

Australian Post-Graduate Award provided to Kerry Hall

- National Health \& Medical Research Council, Australia.

Career Development Fellowship awarded to K O'Grady 
- Queensland Government, Australia.

Smart Futures Fellowship provided to K O'Grady

- National Health \& Medical Research Council, Australia.

Practitioner Fellowship awarded to AB Chang 